Research Paper

\title{
Long non-coding RNA CCDC144NL-AS1 promotes cell proliferation by regulating the miR-363-3p/GALNT7 axis in colorectal cancer
}

Yue Zhang\#, Chaofan Peng\#, Jie Li\#, Dongsheng Zhang, Chuan Zhang, Kangpeng Jin, Dongjian Ji, Wen Peng, Junwei Tang, Yifei Feng ${ }^{\bowtie}$ and Yueming Sun ${ }^{\bowtie}$

Department of General Surgery, The First Affiliated Hospital of Nanjing Medical University, Nanjing, Jiangsu, China.

\#These authors contributed equally to this study.

$\triangle$ Corresponding authors: Yueming Sun, Department of General Surgery, The First Affiliated Hospital of Nanjing Medical University, Nanjing, Jiangsu 210029, China. Tel.: +86-025-6830-6026; Fax: +86 025-68306028; E-mail: sunyueming@njmu.edu.cn; Yifei Feng, Department of General Surgery, The First Affiliated Hospital of Nanjing Medical University, Nanjing, Jiangsu 210029, China. Tel.: +86-025-6830-6026; Fax: +86 025-68306028; E-mail: fengyifei1982@163.com.

(C) The author(s). This is an open access article distributed under the terms of the Creative Commons Attribution License (https://creativecommons.org/licenses/by/4.0/). See http://ivyspring.com/terms for full terms and conditions.

Received: 2021.08.09; Accepted: 2021.11.30; Published: 2022.01.01

\begin{abstract}
Colorectal cancer (CRC) is a burdensome health concern worldwide. Long non-coding RNA (IncRNA) have emerged as vital roles in multiple cancers, including CRC. Increasing evidence has demonstrated that IncRNA CCDC144NL-ASI acts crucial roles in tumor developments. Nevertheless, its role in CRC remains largely unknown. The level of CCDC144NL-ASI expression was detected in $100 \mathrm{CRC}$ tissues and paired adjacent tissues. The gain- and loss-of-function experiments were conducted to investigate the biological functions of CCDC144NL-ASI in vitro and in vivo. The potential mechanism of CCDC144NL-AS1 exerting as competing endogenous RNAs (ceRNAs) was demonstrated by bioinformatics, luciferase reporter assay and in vitro experiments. CCDC144NL-AS1 was up-regulated in CRC tissues and cells. High CCDC144NL-ASI was connected with the adverse clinicopathological features and worse prognosis of CRC. Furthermore, knockdown of CCDC144NL-AS inhibited the cell proliferation and led to the cell cycle G0-1/S arrest, whereas upregulated CCDC144NL-ASI obtained the inverse results. Further study found that CCDC144NL-AS1 functioned as ceRNAs in regulating CRC proliferation. MiR-363-3p was the target of CCDC144NL-AS1, which sponges GALNT7 in regulating cell growth of CRC. The study demonstrated that the CCDC144NL-AS1/miR-363-3p/GALNT7 axis exerts on key roles in cell proliferation and presents an emerging target for CRC therapy and prognostic biomarker.
\end{abstract}

Key words: CCDC144NL-AS1, miR-363-3p, GALNT7, cell proliferation, colorectal cancer

\section{Introduction}

Colorectal cancer (CRC) is major burdensome health problems worldwide, with an evaluative $1,800,000$ cases diagnosing and 0.8 million cases dying in 2018 [1]. In China, CRC also raises a critical health concern for its high incidence and mortality rate [2]. In spite of the tremendous progress achieved in the treatment of CRC under great attention $[3,4]$, the complex molecular regulatory mechanisms of CRC are still unclear. Hence, it is crucial to further explore the unknown mechanisms of CRC progression.

Long non-coding RNAs (lncRNAs) are noncoding RNA consisting of $>200$ nucleotides with limited or no protein-coding ability [5]. It has been found lncRNA abundantly distributed in the nucleus and cytoplasm of mammalian and plant cells $[6,7]$. These lncRNAs can participate in multiple cellular processes, including cell growth, metastasis and differentiation $[8,9]$. Their regulation of genes is common at epigenetic, transcriptional and post transcriptional levels [10, 11]. Increasing evidence showed that IncRNA can play the crucial role in a variety of tumors, including CRC [12-14]. CCDC144NL-AS1 was a lncRNA discovered in recent years. Despite few reports about it, CCDC144NL-AS1 was investigated to act key roles in the multiple biological regulation, like positive regulation of 
endometriosis and inhibition of naïve-like state conservation of human pluripotent stem cells $[15,16]$. It has also been reported to act as an oncogene in gastric cancer [17], osteosarcoma [18] and ovarian cancer [19]. In CRC, CCDC144NL-AS1 was predicted that could be involved in the copy number variations, which contributed to the poor prognosis of patients [20]. However, the expression and function of CCDC144NL-AS1 in CRC are still unknown.

In this study, CCDC144NL-AS1was first detected and analyzed in CRC. Then we further explored the biological roles and mechanisms of CCDC144NL-AS1 in cell proliferation of CRC. All data suggested that CCDC144NL-AS1 is a tumor inhibitor of CRC and may be promising biomarker for diagnosis and molecular therapeutic targets in CRC.

\section{Material and methods}

\section{Clinical specimen}

Total 100 CRC tissues and adjacent tissues were obtained from the First Affiliated Hospital of Nanjing Medical University (NMU). All cases were between 18 and 75 years old, and were confirmed as primary colorectal adenocarcinoma by postoperative pathology. Patients who had received radiotherapy or chemotherapy before operation were excluded. All samples were frozen at $-80{ }^{\circ} \mathrm{C}$ for long-term preservation. All patients signed informed consent before specimen collection. This research was ratified by the Ethics Committee of NMU.

\section{Cell lines and culture}

Five CRC cell lines (HCT116, LoVo, SW480, DLD-1 and HT29) and normal intestinal epithelial cell (NCM460) were obtained from the Cell Bank of Type Culture Collection of the Chinese Academy of Sciences (Shanghai, China). SW480 and DLD-1 were cultured in RPMI 1640 (Winsent, Canada); HCT116, LoVo, HT29 and NCM460 were maintained in Dulbecco's modifed Eagle's medium (Winsent, Canada). All the medium replenished with $10 \%$ fetal bovine serum in a 5\% CO2 humidified incubator at 37 ${ }^{\circ} \mathrm{C}$.

\section{qRT-PCR and RT-PCR of microRNA}

The procedures were referred to the publication [21]. The specific primer for qRT-PCR and RT-PCR used in the study were shown in supplementary Table 1.

\section{Small interfering RNA transfection and lentiviral vectors}

Inhibition and overexpression of CCDC144NLAS1 was conducted by using a plasmid synthesized in GeneChem Corporation (Shanghai, China), and the empty vector was used as the negative control. Transfections of siRNA were carried out using Lipofectamine3000 (Invitrogen, USA) following the manufacturer's instructions, and their transfection efficiency were analyzed after 48 hours by qRT-PCR. A lentivirus expressing a small hairpin RNA (shRNA) against CCDC144NL-AS1 and negative control shRNA were designed by GeneChem Corporation (Shanghai, China). Puromycin was used to select for stable clones after 48 hours.

\section{Western blotting}

Procedures were as described previously [21]. The information about all antibodies used in the study is as follows: GALNT7 (Abcam, ab254971), GAPDH (Abcam, ab181602), cyclin-dependent kinase 4 (CDK4) (Abcam, ab108357), Cyclin D1 (Abcam, ab16663), p27 (Abcam, ab32034).

\section{Cell viability assay}

The Cell Counting Kit- 8 (CCK- 8 ) (Beyotime, Shanghai, China) reagent was added into the cells for $120 \mathrm{~min}$, then detect cell viability. $450 \mathrm{~nm}$ wavelength was chosen to detect.

500 differentially treated cells were added in a 6-well plate. The detailed procedures were adopted from the publication [22].

\section{5-Ethynyl-2'-deoxyuridine (Edu)}

Detailed procedures were described in previous publication [22]. Nikon microscope (Nikon, Japan) captured the images.

\section{RNA fluorescent in situ hybridization (FISH)}

The paired FISH kit (RiboBio, China) and the probe of CCDC144NL-AS1 were designed and synthesized by RiboBio (Guangzhou, China). Images were snapped by a confocal microscopy.

\section{Cell cycle}

The treated cells were supplemented with $75 \%$ ethanol at $4{ }^{\circ} \mathrm{C}$ at least $12 \mathrm{~h}$. Before detection, cells were washed twice using PSB after the separated ethyl alcohol removed, and then were stained with corresponding cell cycle detection reagents for $30 \mathrm{~min}$ in avoid of light. Finally, the cell cycle distribution was analyzed by FACSCalibur flow cytometer with CellQuest software (BD Biosciences).

\section{Dual-Luciferase reporter assay}

The procedures were performed as before described [21]. The wild-type and mutant CCDC144NL-AS1/GALNT7 sequences and 3'-UTR of miR-363-3p were synthesized by GeneScript (Nanjing, China). 


\section{Animal experiments}

The animal study was ratified by the Animal Ethics Committee of NMU. For the tumorigenicity studies, different pretreated LoVo and DLD-1 cells were subcutaneously injected into the 4-week old BALB/c nude mice. Every five days, tumor volume was measured. A month later, the tumor tissues were surgically removed after mice euthanasia, then the tumor weight and the expression level of Ki-67 were detected.

\section{Immunohistochemistry (IHC)}

The experimental process of IHC was previously described [23]. The paraffin-embedded tissue sections were deparaffinized in xylene, rehydrated in alcohol and distilled water. Then, the sections were washed in phosphate-buffered saline, placed in $\mathrm{H}_{2} \mathrm{O}_{2}$ in the dark, soaked in $5 \%$ bovine serum albumin. Finally, the tissue sections were reacted with anti-Ki67 antibodies (Abcam, USA) overnight, followed by incubation with secondary antibody conjugated with HRP. The sections were observed with an inverted microscope (Nikon, Japan). The Ki-67 staining intensity was graded as 0 (no staining), 1 (weak), 2 (moderate), and 3 (strong). The proportion of Ki-67 positive cells was scored as $0(<10 \%), 1(10-50 \%), 2(>50 \%)$. Score was calculated as positive rate score multiplied intensity score.

\section{Statistical analysis}

SPSS 22.0 and GraphPad Prism 8.0 were used to analyze the data. Each experiment was conducted in tripe. Student's t-test, Chi-square test and KaplanMeier analysis were used to conduct the data analysis and survival comparison. $P<0.05$ was significant.

\section{Results}

\section{CCDC144NL-AS1 elevated in CRC and correlated with adverse clinical features}

We first analyzed CCDC144NL-AS1 in TCGA database, which contained data of 471 colon adenocarcinoma (COAD) samples and 41 normal tissue samples. The result suggested that CCDC144NL-AS1 increased both in unpaired tissues (Fig. 1A) and paired tissues (Fig. 1B) of COAD in TCGA database. Then, 100 CRC tissues and adjacent tissues was used to confirm the level of CCDC144NL-AS1 expression by qRT-PCR, and results showed that CCDC144NL-AS1 was increased in CRC tissues in comparison with adjacent tissues (Fig. 1C). Further detection presented that CCDC144NL-AS1 was also elevated in CRC cell lines in compared with colon epithelial mucosa cell line NCM460 (Fig. 1D). Due to CCDC144NL-AS1 in LoVo and DLD-1 was in the middle expression of all CRC cell lines, they were chosen for subsequent study. By further analyzing the relationship between CCDC144NL-AS1 expression and clinicopathological information, we discovered that higher CCDC144NLAS1 predicted worse clinical pathological features (Table 1). Moreover, the online Kaplan Meier plot (GEPIA) indicated that a higher CCDC144NL-AS1 expression level predicted poorer overall survival in COAD (Fig. 1E). Based on follow-up information of the 100 patients, we found that upregulation of CCDC144NLAS1 was connected with worse overall survival (OS) and disease-free survival (DFS) in CRC by using Kaplan-Meier curves (Fig. $1 F$ and G). Abovementioned results showed that CCDC144NLAS1 possibly played an oncogenic role in CRC.

Table 1. Expression of CCDC144NL-AS1 in CRC tissues and adjacent tissues

\begin{tabular}{|c|c|c|c|c|}
\hline \multirow[t]{2}{*}{ Clinical features } & \multicolumn{4}{|c|}{ CCDC144NL-AS1 expression } \\
\hline & $\mathrm{n}$ & high & low & P value \\
\hline Gender & & 50 & 50 & \\
\hline $\operatorname{man}$ & 62 & 32 & 30 & 0.680 \\
\hline female & 38 & 18 & 20 & \\
\hline \multicolumn{5}{|l|}{ Age (year) } \\
\hline$>60$ & 65 & 30 & 35 & 0.295 \\
\hline$\leq 60$ & 35 & 20 & 15 & \\
\hline \multicolumn{5}{|l|}{ Tumor size } \\
\hline$>5 \mathrm{~cm}$ & 42 & 26 & 16 & $0.043^{*}$ \\
\hline$\leq 5 \mathrm{~cm}$ & 58 & 24 & 34 & \\
\hline \multicolumn{5}{|c|}{ Depth of invasion } \\
\hline T1/T2 & 35 & 12 & 23 & $0.021^{*}$ \\
\hline $\mathrm{T} 3 / \mathrm{T} 4$ & 65 & 38 & 27 & \\
\hline \multicolumn{5}{|c|}{ Lymph node metastasis } \\
\hline Absent & 39 & 14 & 25 & $0.024^{*}$ \\
\hline Present & 61 & 36 & 25 & \\
\hline \multicolumn{5}{|l|}{ Liver metastasis } \\
\hline Present & 10 & 7 & 3 & 0.182 \\
\hline Absent & 90 & 43 & 47 & \\
\hline \multicolumn{5}{|l|}{ Location } \\
\hline rectal & 58 & 28 & 30 & 0.685 \\
\hline colon & 42 & 22 & 20 & \\
\hline
\end{tabular}

\section{Knockdown of CCDC144NL-AS1 inhibits cell proliferation of $C R C$ in vitro}

CCDC44NL-AS1 were knocked down in LoVo and DLD-1 cells with a small interfering RNA (si-CCDC44NL-AS1), respectively. The qRT-PCR results presented that CCDC144NL-AS1 were significantly inhibited by si-CCDC144NL-AS1 both in LoVo and DLD-1 cells (Fig. 2A). Then the CCK-8 presented that downregulated CCDC144NL-AS1 markedly decreased the cell viability (Fig. 2B and 2C). As showed in Fig. 2D and 2E, silencing CCDC144NLAS1 repressed the cell formative abilities of LoVo and DLD-1. The Edu assays confirmed the similar trends that knockdown CCDC144NL-AS1 dramatically inhibited the DNA synthesis process of LoVo and 
DLD-1 cells (Fig. 2F-H). Furthermore, the results of cell cycle distribution revealed that knockdown of CCDC44NL-AS1 could result in cell cycle arrest in G0-1/S phage (Fig. 2I-K).

\section{Overexpression of CCDC144NL-AS1 promotes cell proliferation of CRC}

To verify the function of CCDC144NL-AS1 in CRC cell proliferation, CCDC144NL-AS1 overexpressing plasmids were transfected to LoVo and DLD-1 cells, and result of qRT-PCR indicated that CCDC144NL-AS1 was dramatically elevated (Fig. 3A). Then, cell proliferation assays found that the results of CCDC144NL-AS1 overexpression and knockdown were completely opposite. The CCK-8 assays revealed that upregulation of CCDC144NLAS1 markedly promoted cells viability compared with control vector (Fig. 3B and C). Then the assays of colony formation showed that overexpressed CCDC144NL-AS1 improved the cells formative abilities of LoVo and DLD-1 (Fig. 3D and E). The Edu assay suggested that elevated CCDC144NL-AS1 promoted DNA synthesis process in LoVo and DLD-1 cells (Fig. 3F-H). Cell cycle analysis showed that overexpression of CCDC144NL-AS1 accelerated cells from G0/G1 phase stepping to $S$ phase (Fig. 3I-K). All results demonstrated that CCDC144NL-AS1 promoted LoVo and DLD-1 growth of CRC possibly by $\mathrm{G} 0-1 / \mathrm{S}$ cell cycle regulation.
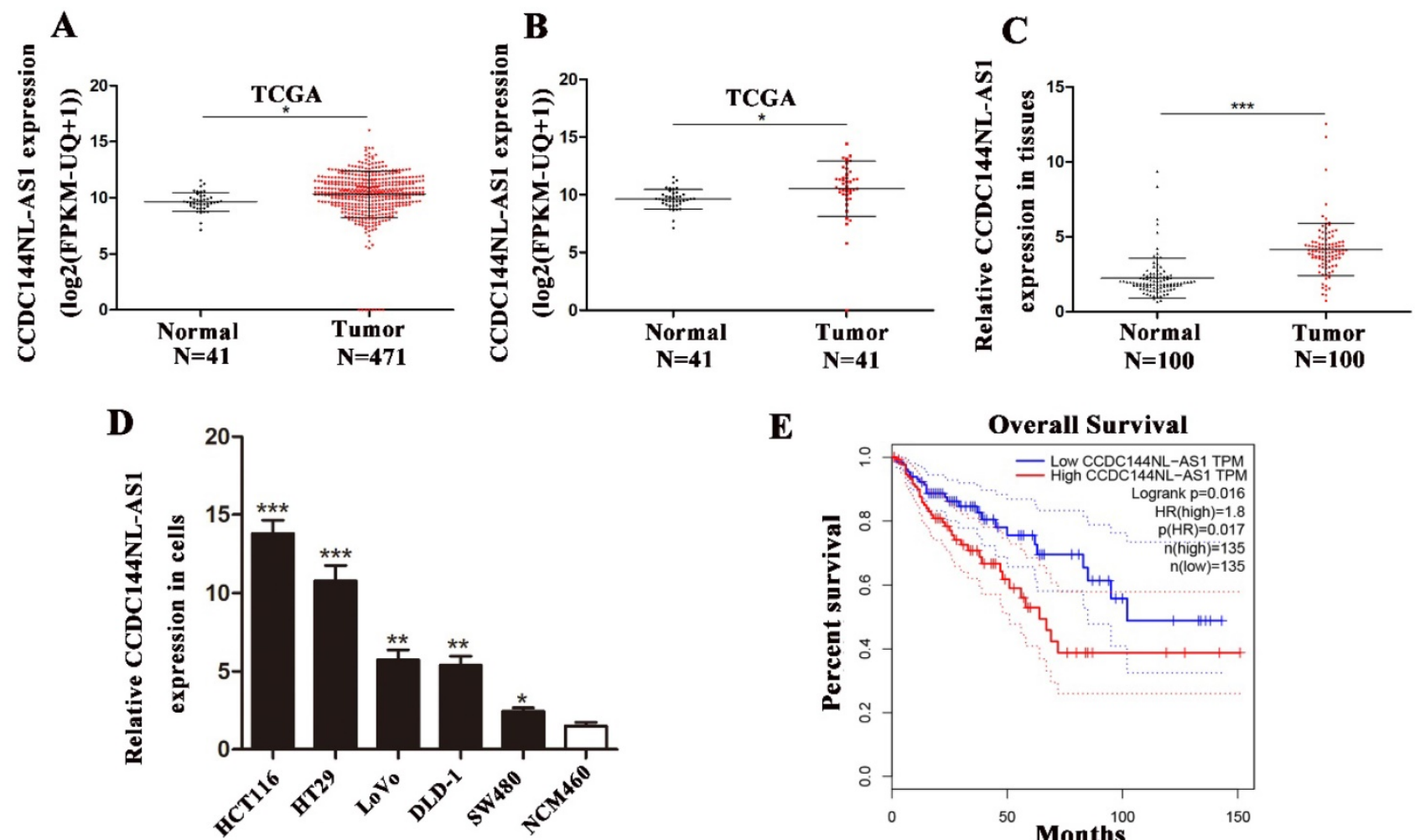

$\mathbf{E}$
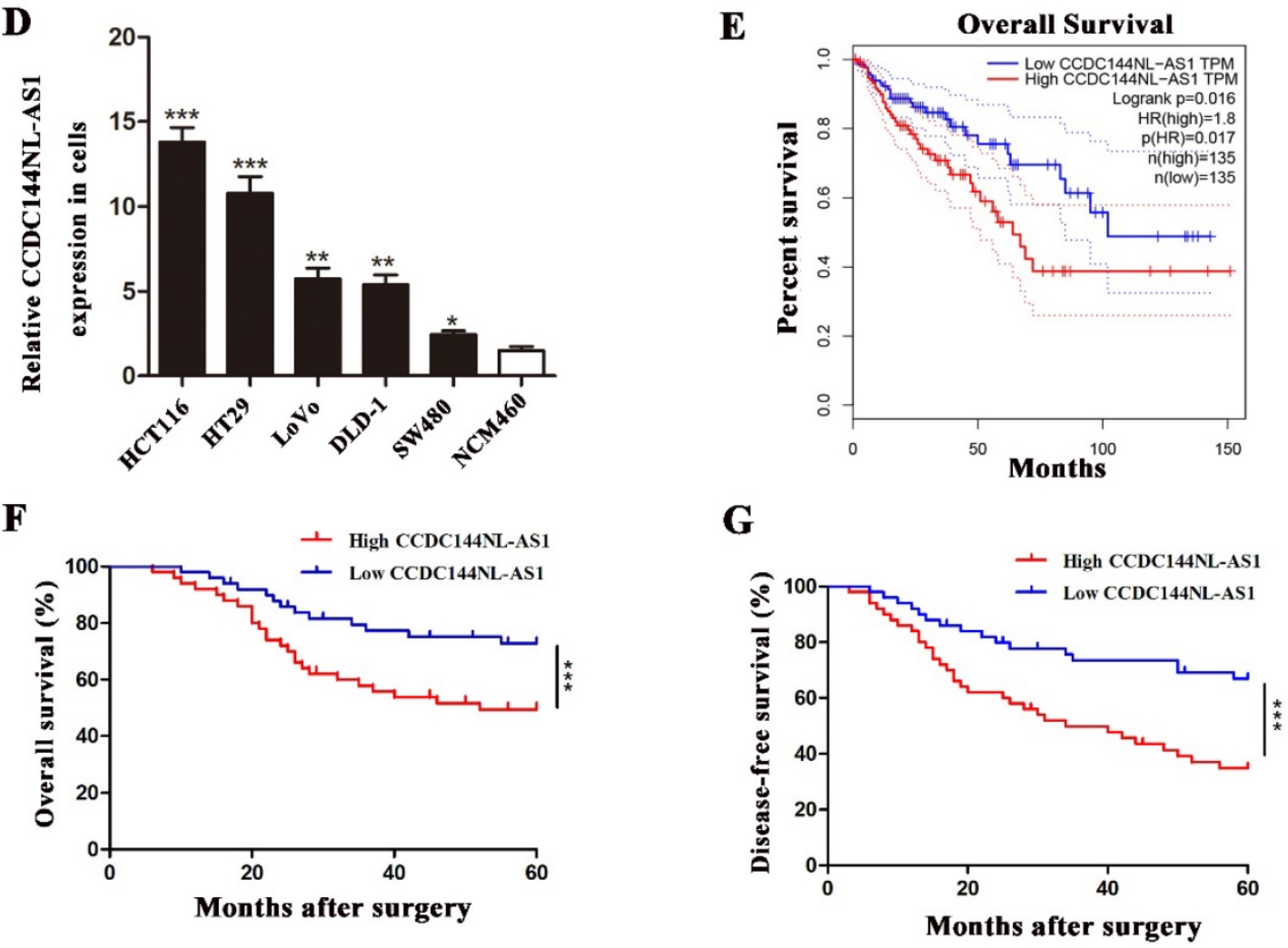

Figure 1. CCDC144NL-AS1 was upregulated in CRC and predicted poor prognosis. A and B. Relative expression of CCDC144NL-AS1 in unpaired and paired COAD from the TCGA database. C. Relative expression of CCDC144NL-AS1 in $100 \mathrm{CRC}$ tissues and matched adjacent normal tissues. D. Relative expression of CCDC144NL-AS1 in CRC cells and normal colon epithelial mucosa cell line (NCM460). E. Online Kaplan-Meier overall survival (OS) curves based on the CCDC144NL-AS1 expression in COAD. F and G. OS and disease-free survival (DFS) of 100 CRC patients based on CCDC144NL-AS1 expression by Kaplan-Meier survival analysis. *p < 0.05 , **p $<0.01, * * * \mathrm{p}<0.001$. 


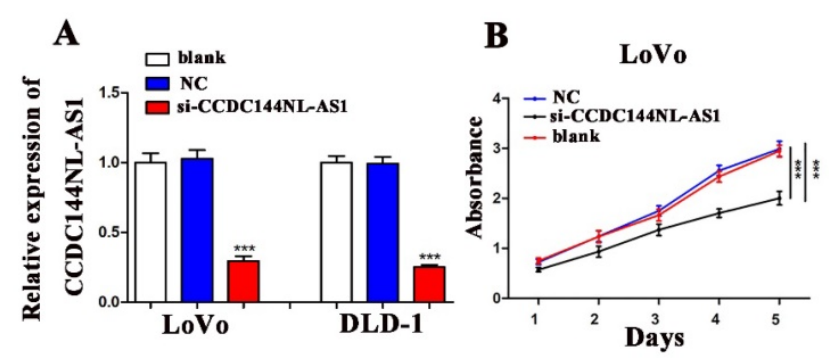

C

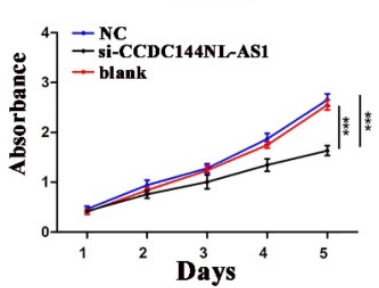

G

F

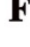

LoVo

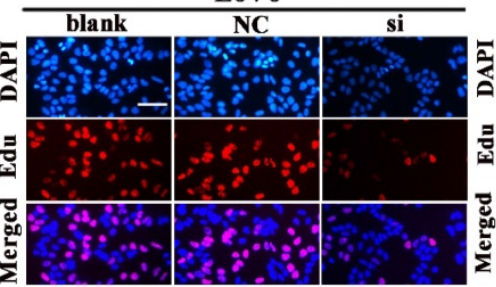

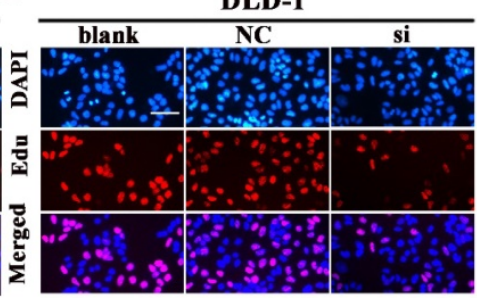

D

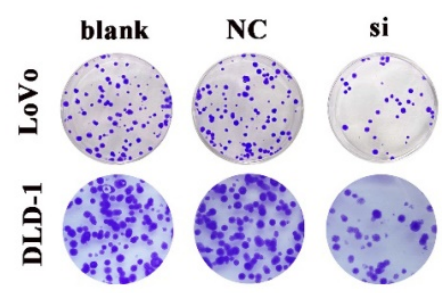

$\mathbf{H}$

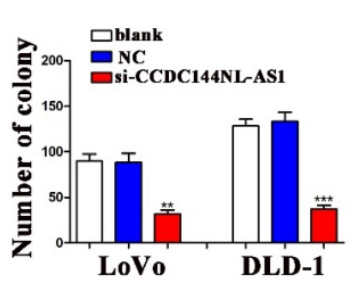

I

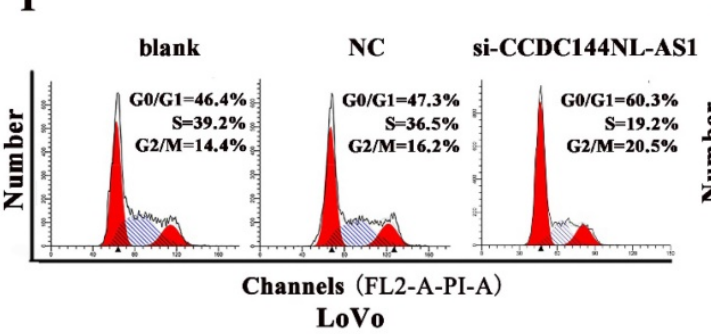

$\mathbf{J}$

\section{$\mathbf{J}$}
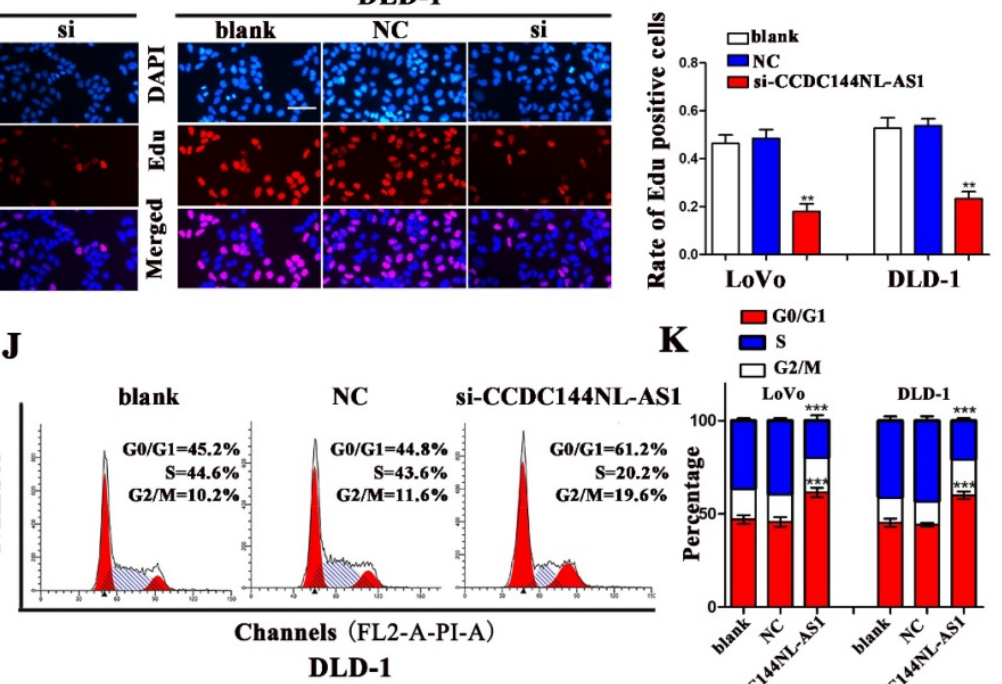

K $\mathrm{s}$

$\square \mathbf{G 2} / \mathbf{M}$

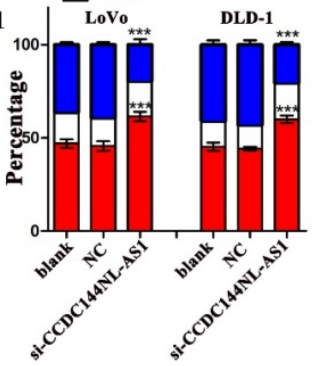

Figure 2. Knockdown of CCDC144NL-AS1 inhibited CRC cell proliferation and caused cell cycle G0-1/S arrest. A. Relative CCDC144NL-AS1 expression in LoVo and DLD-1 cells treated with si-CCDC144NL-AS1 transfection. B and C. Effects of CCDC144NL-AS1 silencing on the cell viability were detected by CCK-8 assay in LoVo and DLD-1 cells. D-H. Effects of CCDC144NL-AS1 silencing on cell proliferation were measured by the colony formation assay and the Edu assay in LoVo and DLD-1 cells. Scale bar: $50 \mu \mathrm{m}$. I-K. Effects of CCDC144NL-ASI silencing on cell cycle distribution in LoVo and DLD-1 cells. $* * p<0.01, * * * p<0.001$.

\section{Knockdown of CCDC144NL-AS1 represses tumor growth in vivo}

We performed the tumor xenografting in nude mice to investigate the effects of CCDC144NL-AS1 in tumorigenesis in vivo. After transfection with CCDC144NL-AS1 inhibitor lentivirus (sh-CCDC144NL-AS1), the mRNA expression of CCDC144NL-AS1 were significantly downregulated (Fig. 4A). We found that the tumor weight and tumor size were markedly decreased with knockdown of CCDC144NL-AS1 both in LoVo and DLD-1 cells (Fig. 4B-F). Furthermore, we detected the expression of $\mathrm{Ki}-67$ in mouse tissues by IHC. The result showed that tumors transfected with sh-CCDC144NLAS1 showed lower expression of Ki-67 in comparison with sh-NC groups (Fig. 4G and $4 \mathrm{H}$ ). These results further indicated CCDC144NL-AS1 acts vital carcinogenic roles in the cell proliferation of CRC in vivo.

\section{CCDC144NL-AS1 exerts on a molecular sponge for miR-363-3p of CRC}

To investigate the potential mechanism by which CCDC144NL-AS1 influences the proliferation of CRC cells, we used CPAT tool to find that CCDC144NLAS1 was mainly located in cytoplasm of human species (Fig. 5A). Then the FISH and qRT-PCR assays further validated that CCDC144NL-AS1 was located in CRC cytoplasm (Fig. 5B and 5C). Therefore, we speculated that CCDC144NL-AS1 possibly served as a ceRNA of miRNAs. Through bioinformatics analysis, we found that CCDC144NL-AS1 may be sponge of miR-363-3p. The results of qRT-PCR showed that miR-363-3p was upregulated after CCDC144NL-AS1 silencing (Fig. 5D), while was downregulated after CCDC144NL-AS1 overexpression (Fig. 5E). Then the mRNA expression of miR-363-3p was detected by qRT-PCR in the 100 paired of CRC tissues, and the results showed that it was significantly downregulated in tumor tissues (Fig. 5F). Further analysis revealed that a negative correlation existed between CCDC144NL-AS1 and miR-363-3p (Fig. 5G). After transfection of miR-363-3p inhibitor (miR-363$3 p-i n)$ and miR-363-3p mimic, the expression of miR-363-3p was significantly downregulated and upregulated, respectively (Fig. 5J and 5K). To examine whether CCDC144NL-AS1 directly targeted miR-363$3 p$, we conducted a luciferase assay in LoVo cells, and 
the result showed that miR-363-3p dramatically repressed the luciferase activity of the wild-type CCDC144NL-AS1 3'UTR (Fig. 5H and 5I). All data revealed that CCDC144NL-AS1 may sponge miR-363-3p in CRC cells.
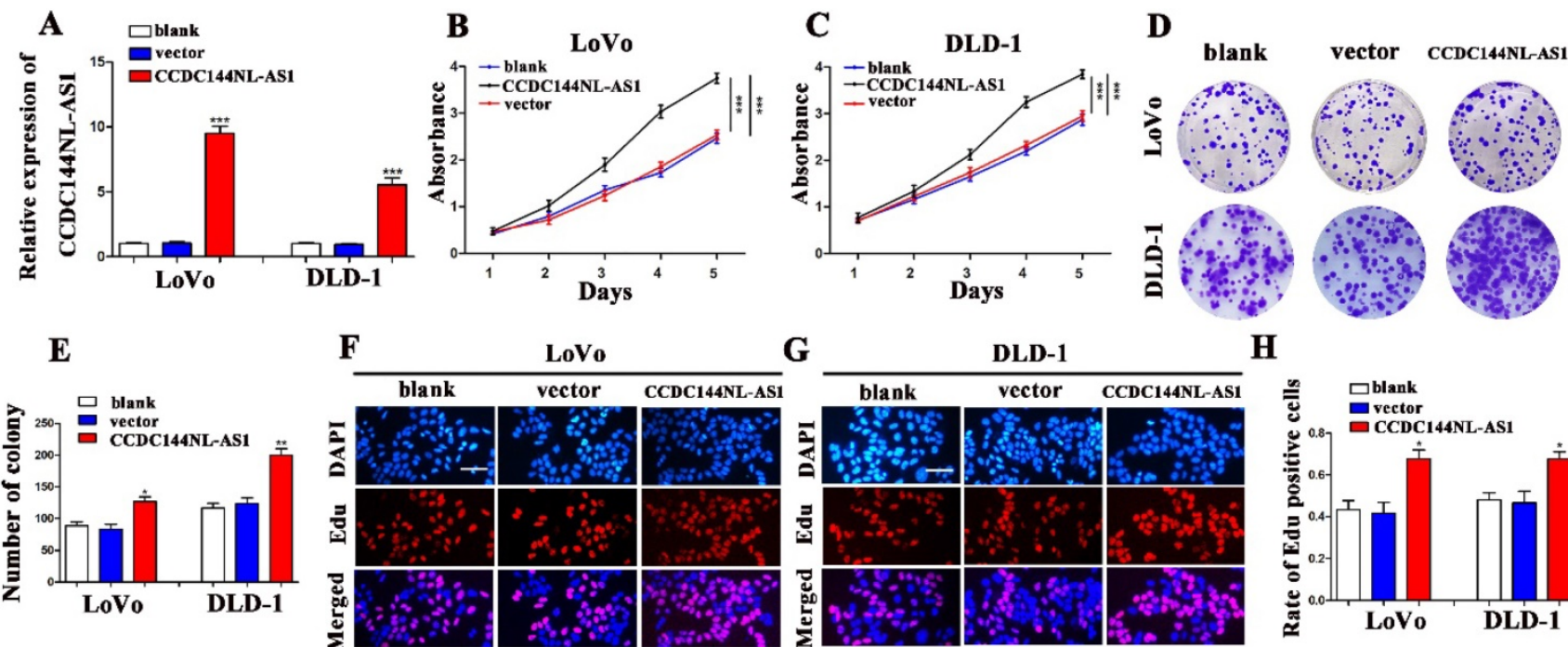

G

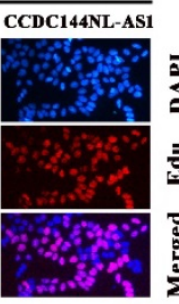

DLD-1

H
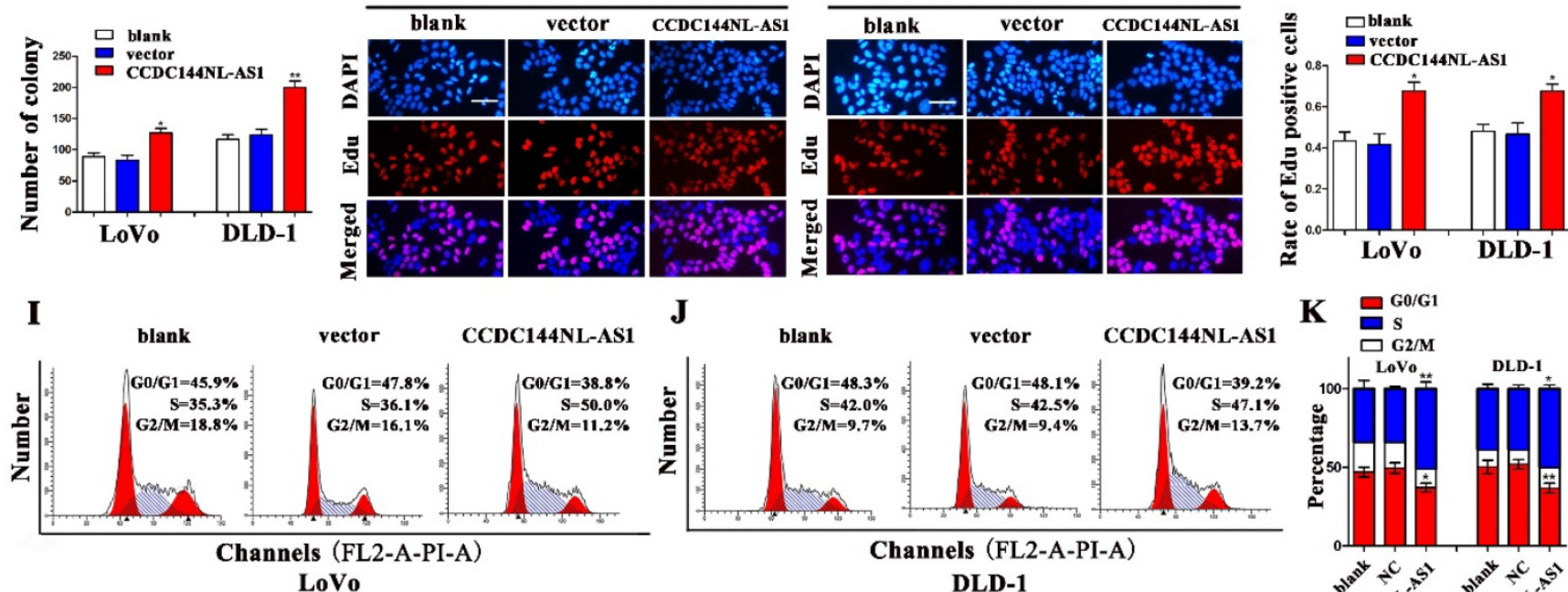

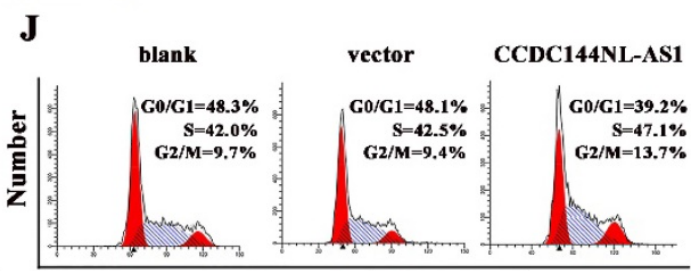

Channels (FL2-A-PI-A)

DLD-1

\section{K $\mathbf{G}_{\mathbf{G} / \mathbf{G} 1}$}

$\mathbf{s}$

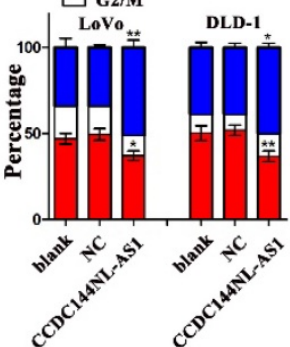

Figure 3. Overexpression of CCDC144NL-AS1 promoted CRC cell proliferation and accelerated cell cycle G0-1/S transition. A. Relative CCDC144NL-AS1 expression in LoVo and DLD-1 cells treated with CCDC144NL-ASI overexpressing plasmid transfection. B and C. Effects of CCDC144NL-ASI overexpression on cell viability were detected by CCK-8 assay in LoVo and DLD-1 cells. D-H. Effects of CCDC144NL-AS1 overexpression on cell proliferation were measured by the colony formation assay and the Edu assay in LoVo and DLD-1 cells. Scale bar: $50 \mu \mathrm{m}$. I-K. Effects of CCDC144NL-AS1 overexpression on cell cycle distribution in LoVo and DLD-1 cells. *p<0.05, **p $<0.01, * * * p<0.001$

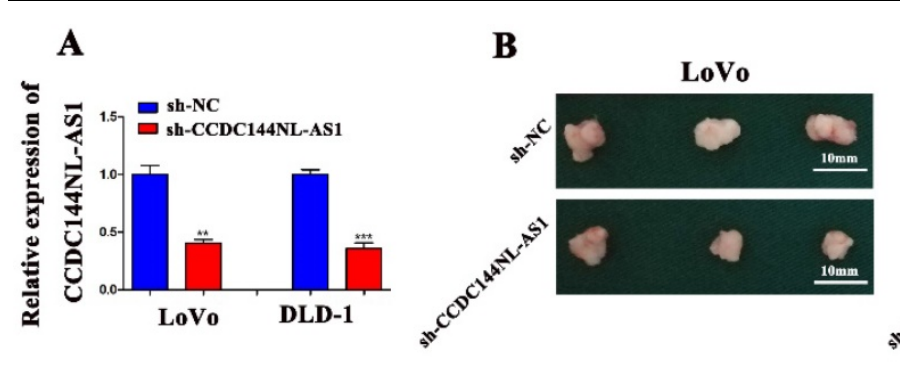

$\mathbf{E}$

LoVo

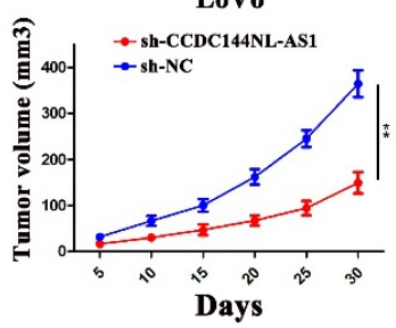

$\mathbf{F}$

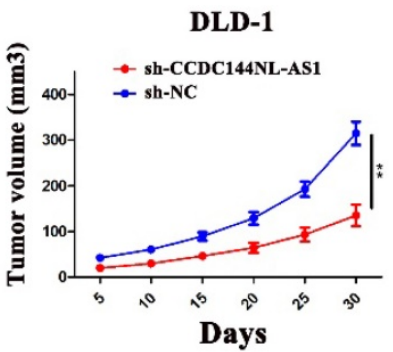

C

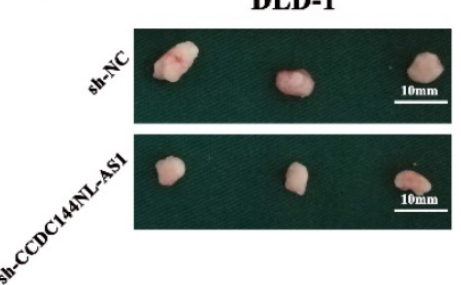

G

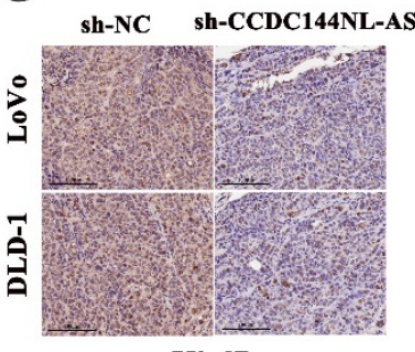

Ki-67
D

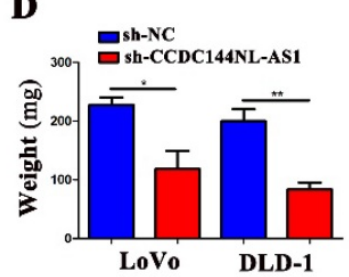

$\mathbf{H}$

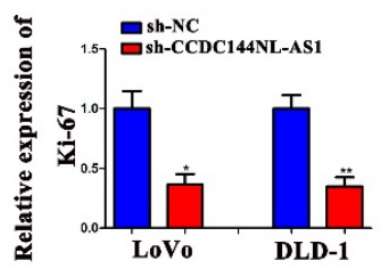

Figure 4. Knockdown of CCDC144NL-AS1 inhibited CRC tumor growth in vivo. A. Relative mRNA expression of CCDC144NL-AS1 in LoVo and DLD-1 cells following sh-CCDC144NL-ASI transfection. B. Images of xenograft tumors in the nude mouse model under different treatments. D-F. Analysis of tumor weight and size in the sh-CCDC144NL-AS1 and control groups. G and $\mathbf{H}$. The expression of Ki-67 from the xenografts were measured by IHC. ${ }^{*} p<0.05, * * p<0.01, * * * p<0.001$. 
$\mathbf{A}$

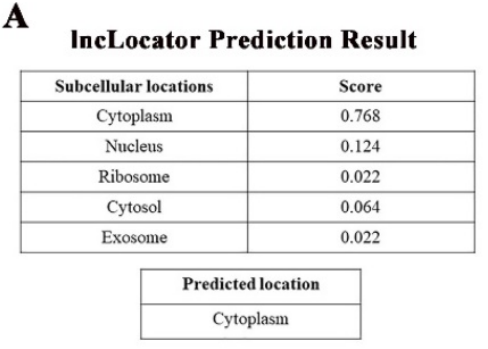

B

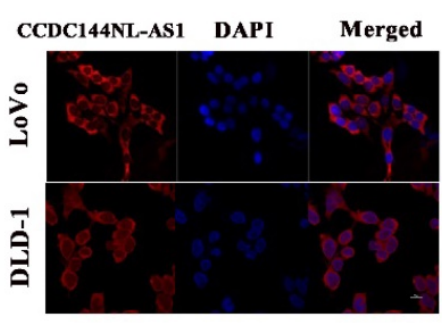

C

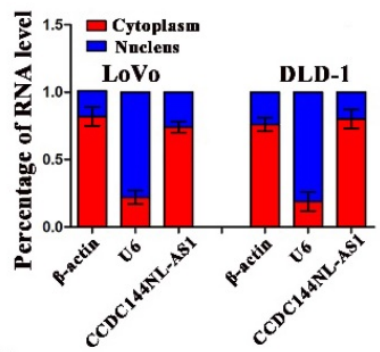

G

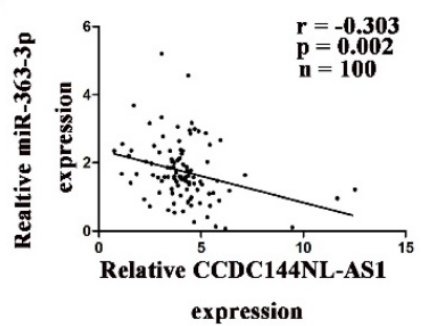

$\mathbf{K}$

$\mathbf{J}$
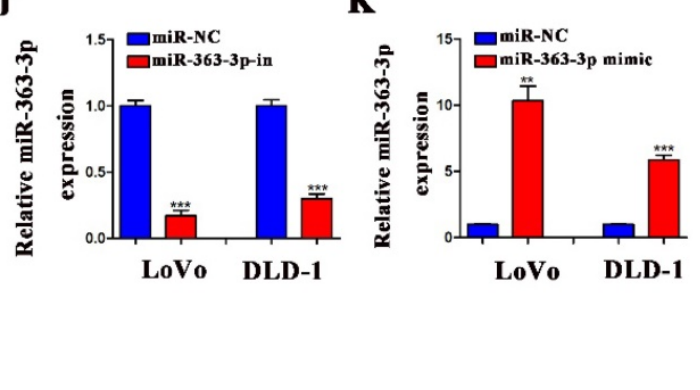

Figure 5. CCDC144NL-AS1 acted as a molecular sponge for miR-363-3p in CRC cells. A. Prediction of CCDC144NL-AS1 localization in cells by bioinformatics tools. B and C. Subcellular localization of CCDC144NL-AS1 in CRC cells detected by FISH and qRT-PCR. D and E. Effects of CCDC144NL-AS1 alteration on the expression of miR-363-3p detected by qRT-PCR in LoVo and DLD-1 cells. F. Relative expression of miR-363-3p in 100 CRC tissues and matched adjacent normal tissues. G. Correlation analysis of the expression of CCDC144NL-ASI and miR-363-3p in 100 CRC tissues. H. The predicted binding sites between CCDC144NL-ASI and miR-363-3p. I. Luciferase activity after MiR-363-3p mimics or miR-NC and pmirGLO-CCDC144NL-AS1-WT or pmirGLO-CCDC144NL-AS1-MUT co-transfected into LoVo cells. J and K. Relative expression of miR-363-3p in LoVo and DLD-1 cells treated with miR-363-3p inhibitor (miR-363-3p-in) and mimic. $* * p<0.01, * * * p<0.001$.

\section{CCDC144NL-AS1 promotes cell proliferation of CRC cells by miR-363-3p regulation}

To reveal whether the carcinogenesis of CCDC144NL-AS1 on CRC cells was mediated by miR-363-3p, LoVo and DLD-1 cells were co-transfected with si-CCDC144NL-AS1/NC and miR-363-3p inhibitor (miR-363-3p-in)/miRNA negative control (miR-NC). Then cell proliferation assays were performed, and the results showed that knockdown of CCDC144NL-AS1 alone significantly inhibited the cell proliferation as the previous outcome, and miR-363-3p silencing alone remarkably promoted the cell proliferation of CRC cells. Moreover, miR-363-3p silencing could dramatically attenuate the function of CCDC144NL-AS1 knockdown on cell proliferation (Fig. 6A-H). These data indicated that CCDC144NL-AS1 promotes cell proliferation on CRC by mediating miR-363-3p.

GALNT7 was a target of miR-363-3p involved in CCDC144NL-AS1 induced cell proliferation

To seek the targets of miR-363-3p in CRC, we used the bioinformatics data by miRDB, miRtarbase, DIANA and Targetscan databases. 25 candidate genes were discovered after the intersection of these databases, GALNT7 was contained (Fig. 7A). Through literature review, we found GALNT7 played an important role on the biological regulation of CRC cells, which was highly likely to be the target of miR-363-3p. To verify our speculation, GALNT7 was detected in these 100 paired CRC tissues and adjacent tissues, and the results presented that GALNT7 was overexpressed in CRC tissues in comparison with the adjacent tissues (Fig. 7B). Further analysis found that a remarkably negative relation between GALNT7 and miR-363-3p (Fig. 7C), and a positive correlation was between CCDC144NL-AS1 and GALNT7 in these 100 tumor tissues (Fig. 7D). Then analysis of luciferase assays confirmed that miR-363-3p remarkably restrained the luciferase activities in the wild type of GALNT7 3'UTR (Fig. 7E and 7F), indicating miR363-3p could sponge GALNT7 in CRC cells. Then we detected the GLANT7 expression in CRC cells, the level of GALNT7 mRNA and protein was both downregulated with the upregulation of miR-363-3p, 
but increased with the downregulation of miR-363-3p (Fig. 7G and 7H). si-GALNT7 was transfected to LoVo and DLD-1 to investigate the relation between GALNT7 and miR-363-3p. GALNT7 was downregulated both on mRNA and protein levels after si-GALNT7 transfection (Fig. 7I and 7J). Then, LoVo and DLD-1 were co-transfected with miR-363-3p-in/miR-NC and si-GALNT7/NC. CCK-8 and Edu assays both revealed that downregulated GALNT7 not only repressed the cell growth, but also remarkably attenuated the function of miR-363-3p knockdown on cell inhibition (Fig. 7K-O). Furthermore, we detected GALNT7, CDK4, Cyclin D1 and p27 in CRC cells by western blot, we found GLANT, CDK4 and Cyclin D1 were downregulated and p27 was upregulated after CCDC144NL-AS1 knockdown. The similar results were also observed after CCD144NL-AS1 overexpression (Fig. 7P). All these results indicated that GALNT7 participated in the regulation of CCDC144NL-AS1 on the cell proliferation of $\mathrm{CRC}$ as the downstream of miR-363-3p, and the complete mechanism by which CCDC144NL-AS1 promoted CRC cell proliferation was shown in Figure 8.

\section{Discussion}

Accumulating investigations revealed that lncRNAs play vital roles in human diseases, including various cancers [24-27]. Through the analysis of TCGA database, we discovered that CCDC144NLAS1 elevated in COAD, and higher CCDC144NL-AS1 expression predicted a poorer prognosis of patients. Our results first detected that CCDC144NL-AS1 was significantly increased in CRC. Upregulated CCDC144NL-AS1 was closely related to the worse clinicopathological features. Furthermore, elevated CCDC144NL-AS1 was connected with worse OS and PFS in CRC patients. Functional assays investigated that decreased CCDC144NL-AS1 repressed cell growth and arrested cell cycle, whereas overexpressed CCDC144NL-AS1 reversed the abovementioned results. Animal experiments further detected that decreased CCDC144NL-AS1 inhibited the cell proliferation. All data suggested that CCDC144NL-AS1 plays carcinogenic roles in CRC.
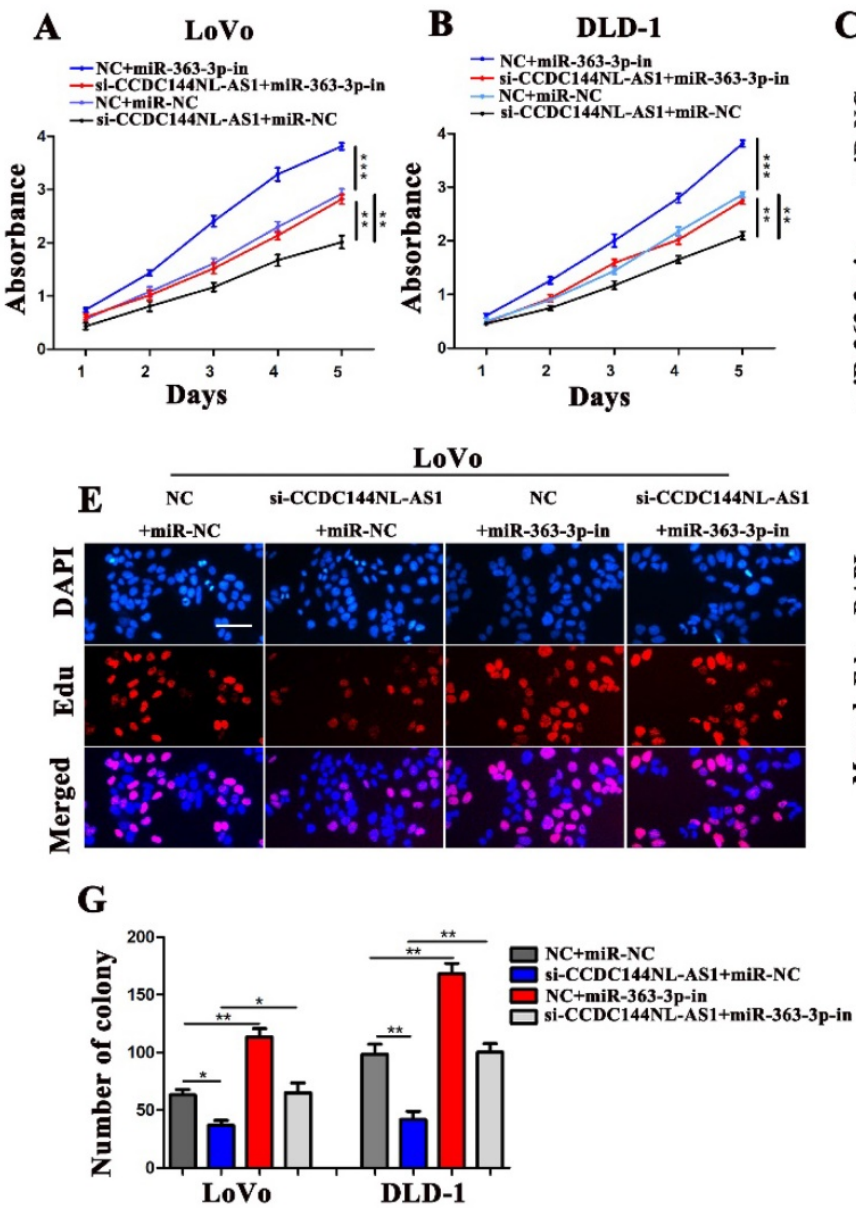
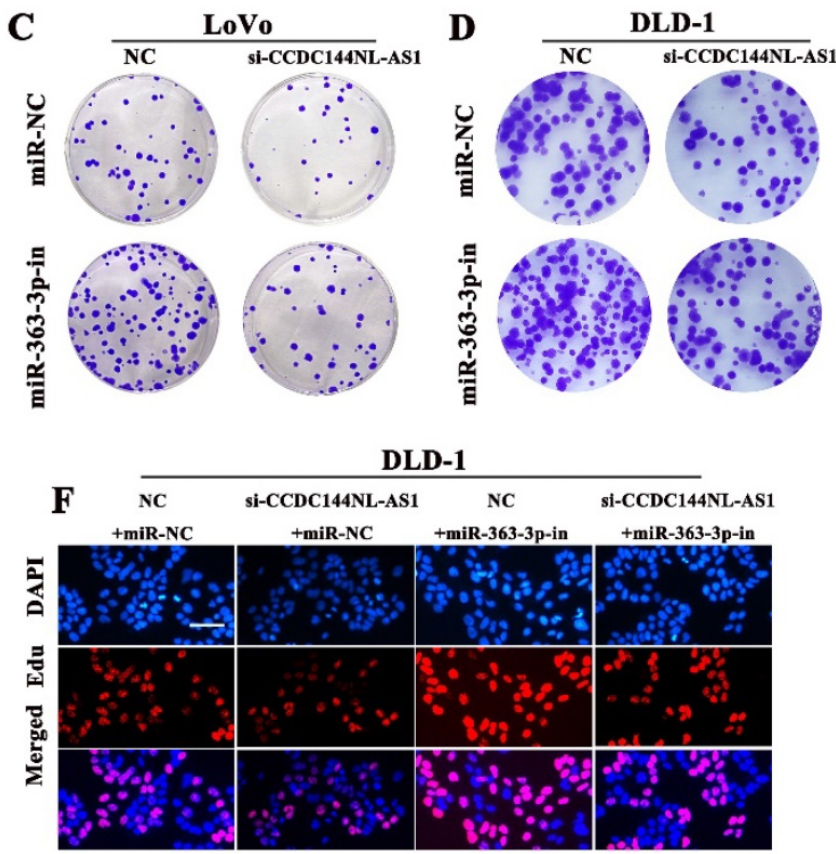

$\mathbf{H}$

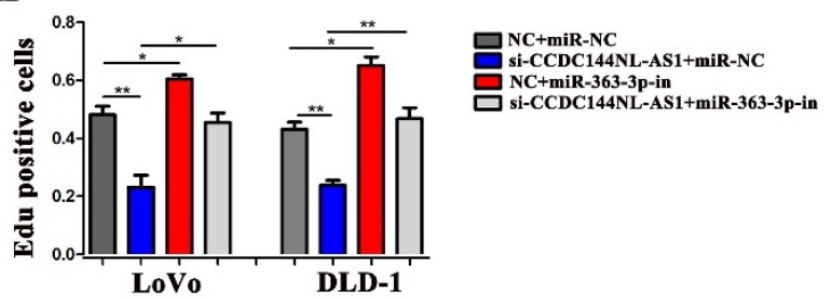

Figure 6. The regulation of CCDC144NL-AS1 on CRC cell was mediated by miR-363-3p. A and B. Cell viability of LoVo and DLD-1 cells in different groups was detected by the CCK-8 assay. C-H. Cell proliferation of LoVo and DLD-1 cells in different groups was detected by the colony formation assay and Edu assay. Scale bar: $50 \mu$ m. $*_{p}<0.05, * * p<0.01, *^{* *} p<0.001$. 

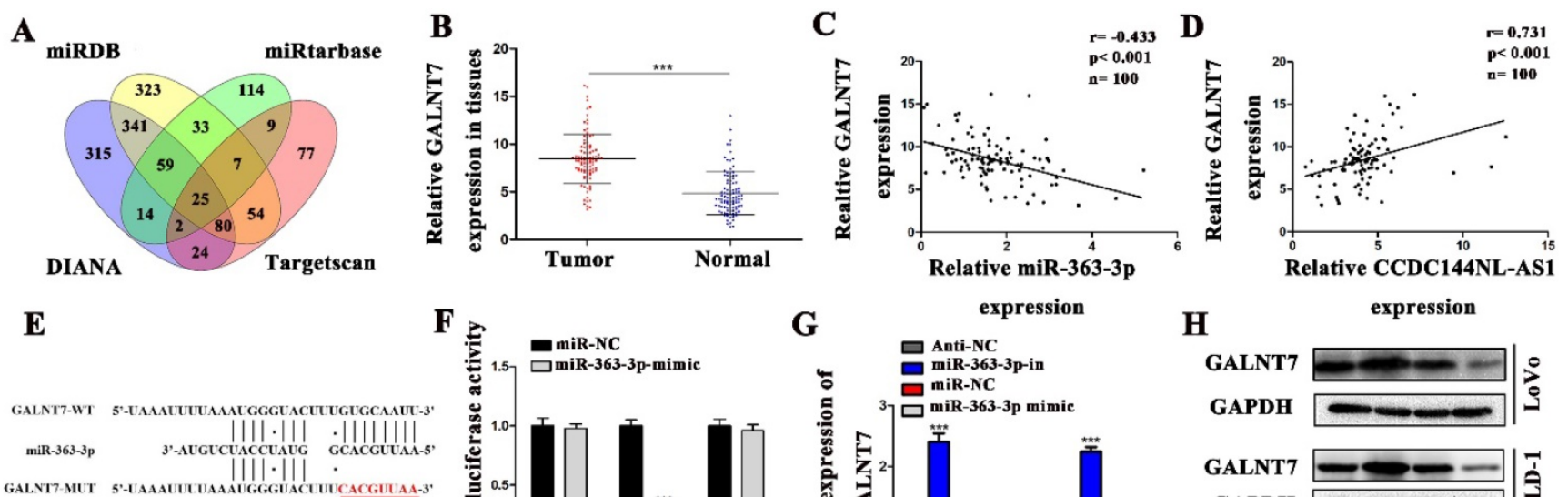

$$
\text { F }
$$

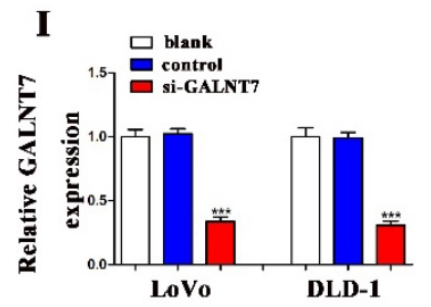

J

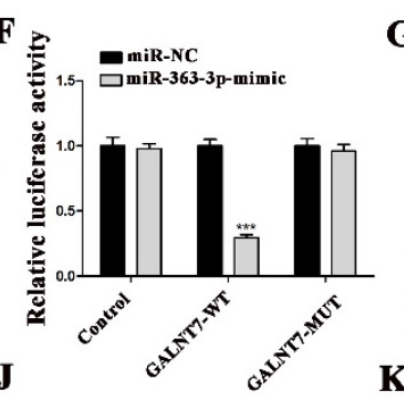

G expression

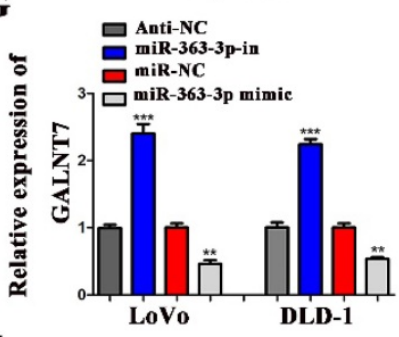

H expression

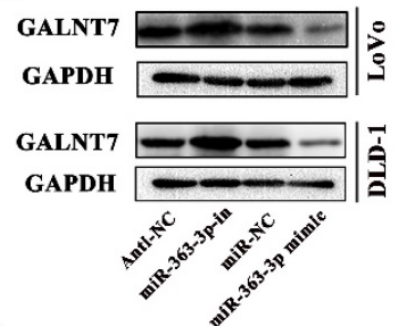

$\mathbf{L}$
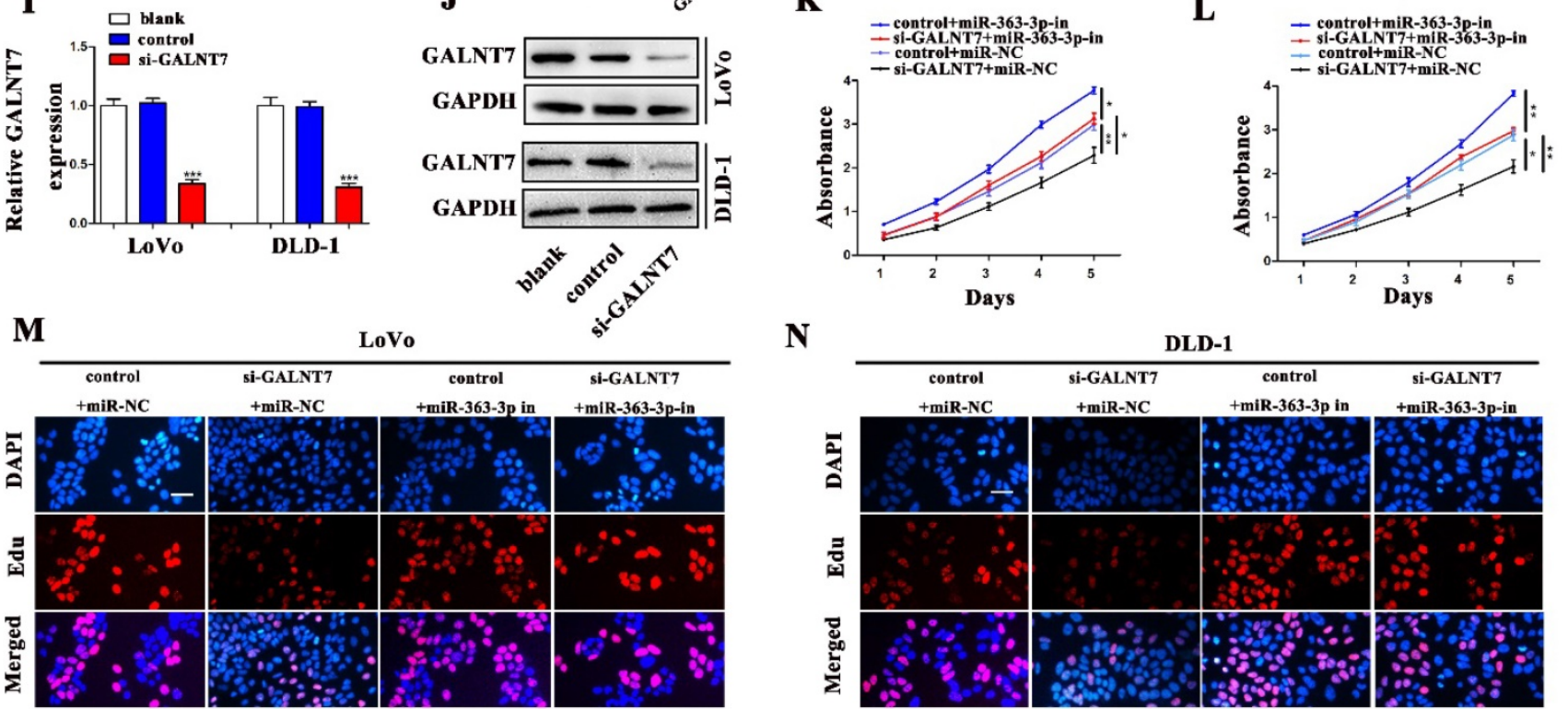

$\mathbf{N}$
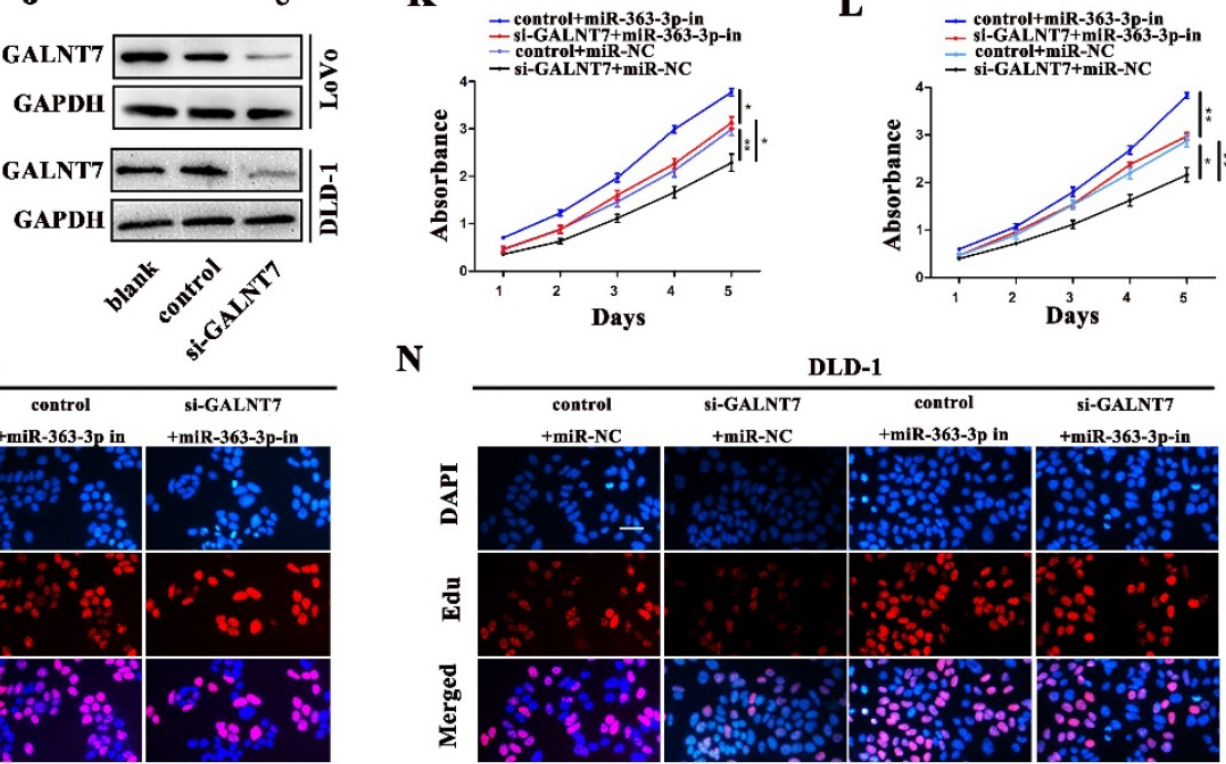

$\mathbf{O}$

$\mathbf{P}$

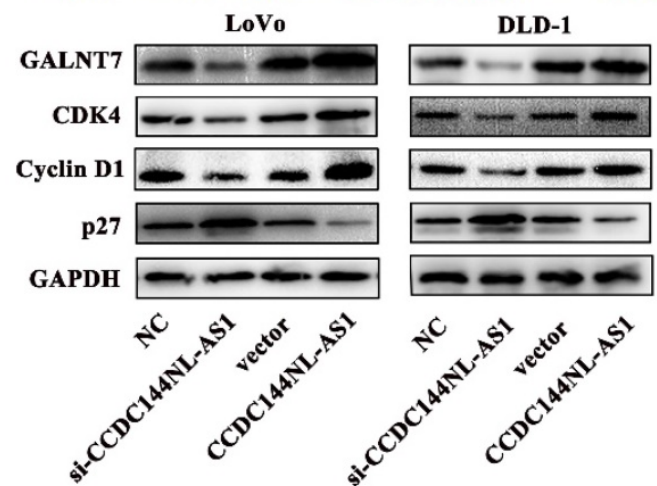

Figure 7. GALNT7, a target gene of miR-363-3p, involved in CCDC144NL-AS1 regulation. A. The potential target genes of miR-363-3p was predicted by miRDB, miRtarbase, DIANA and Targetscan database. B. Relative mRNA expression of GALNT7 in $100 \mathrm{CRC}$ tissues and matched adjacent normal tissues. C. Correlation analysis of the expression of GALNT7 and miR-363-3p in 100 CRC tissues. D. Correlation analysis of the expression of GALNT7 and CCDC144NL-AS1 in 100 CRC tissues. E. The predicted binding sites between GALNT7 and miR-363-3p. F. Luciferase activity after MiR-363-3p mimics or miR-NC and pmirGLO-GALNT7-WT or pmirGLO-GALNT7 -MUT co-transfected into LoVo cells. G and H. GALNT7 expression was detected by qRT-PCR and western blotting after miR-363-3p inhibition and overexpression. I and J. GALNT7 expression was detected by qRT-PCR and western blotting after si-GALNT7 transfection. $\mathbf{K}$ and M. Cell viability of LoVo and DLD-1 cells in different groups was measured by the CCK-8 assay. N and O. Cell proliferation of LoVo and DLD-1 cells in different groups was measured by the Edu assay. Scale bar: $50 \mu \mathrm{m}$. P. The expression of GALNT7, CDK4, Cyclin D1 and p27 was detected in CRC cells by western blotting after CCDC144NL-AS1 knockdown and overexpression. ${ }^{*} p<0.05, * * p<0.01, * * * p<0.001$. 


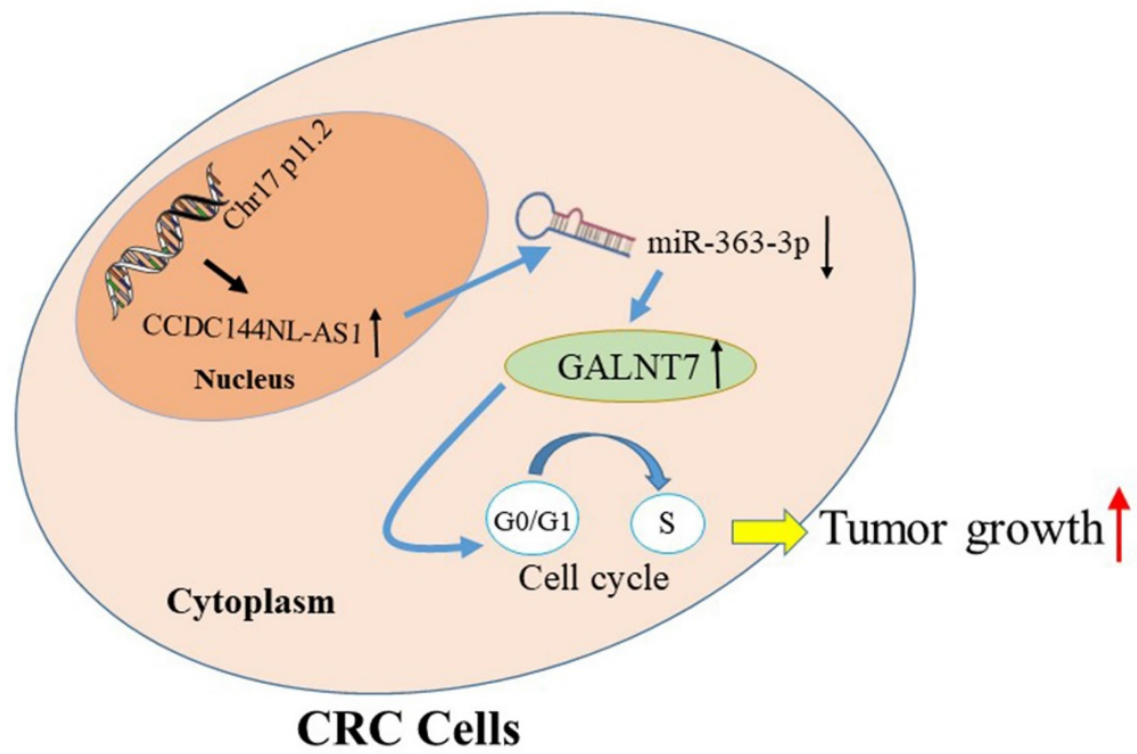

Figure 8. The schematic model of CCDC144NL-ASI in regulating CRC. Increased CCDC144NL-ASI competitively sponged more miR-363-3p, led to the negative effect of miR-363-3p on GALNT7 reduced, then accelerated the cell cycle G0-1/S transition and promoted cell proliferation in CRC cells.

The ceRNAs are transcripts that can regulate each other at post-transcription by competing for shared miRNAs, which was named since 2011 [28, 29]. Sufficient evidence has showed that the functionalities of lncRNAs in tumorigenesis could be partially mediated by ceRNA crosstalk, including CRC $[30,31]$. For example, the oncogenic role of $\mathrm{H} 19$ was reported that attributed to its ceRNA activity to sponge miR-138 and miR-200a in CRC [32, 33]. LncRNA UICLM was also founded that facilitated cell metastasis by serving as ceRNAs of CRC [34]. Recently, the ceRNA role of CCDC144NL-AS1 has been discovered in gastric cancer [17]. In combination with our result that CCDC144NL-AS1 mainly distributed in cytoplasm, we assumed that CCDC144NL-AS1 functions as ceRNAs in CRC. miR-363-3p may be the novel target of CCDC144NL-AS1 through serial assays. miR-363-3p mainly acted as a tumor inhibitor, including ovarian cancer [35], osteosarcoma [36], lung cancer [37], hepatocellular carcinoma [38] and CRC [39]. It was involved in tumorigenesis, cell proliferation, invasion and metastasis by different regulatory mechanisms. Recently, its biological role of ceRNA was also determined in multiple cancers $[35,40]$. However, the relation between CCDC144NL-AS1 and miR-363-3p were unclear in CRC. MiR-363-3p was markedly decreased in CRC tissues, and negative correlations appeared between CCDC144NL-AS1 and miR-363-3p. In vitro results further verified that downregulated miR-363-3p dramatically attenuated the effects of downregulated CCDC144NL-AS1 on CRC cell inhibition. All results demonstrated that CCDC144NL-AS1 plays crucial roles by binding
miR-363-3p in CRC.

The ceRNAs of lncRNAs mainly relies on the derepression of miRNAs' targets [41]. The targets of miRNA are an indispensable part of ceRNA network. We discovered that GalNAc-transferase-7 (GALNT7) was the potential target of miR-363-3p through analysis and screening. Previous studies have demonstrated that aberrant expression of GALNT7 were involved in the developments of multiple tumors, such as glioma [42], cervical cancer [43] and CRC [44], by affecting cell proliferation, differentiation, invasion and migration. GALNT7 was also reported to be targeted by some miRNAs in various cancers, such as miR-123-5p and miR-154 [43, 45]. Moreover, GLANT7 has been reported to be involved in the ceRNA network as the target of miR-34a in CRC [44, 46]. However, the relation between GALNT7 and miR-363-3p was never mentioned. In this study, we discovered GLANT7 was upregulated in CRC, which was accordance with previous literature. Further analysis presented that GALNT7 was positively connected to CCDC144NLAS1, and negatively related to miR-363-3p. Moreover, in vitro results revealed that GLANT knockdown significantly attenuated the effects of downregulated miR-363-3p on CRC cell proliferation. Thus, these results suggested that GALNT7 may be the downstream of miR-363-3p, and the CCDC144NLAS1/miR-363-3p/GALNT7 axis could promote cell proliferation in CRC. Furthermore, accelerated cell proliferation is usual linked to the aberrant regulation of cell cycle [47]. During the whole process of cell cycle, G1/S transition was the crucial point that determines whether cells enter the process of 
proliferation [48]. In the current study, we found that CCDC144NL-AS1 downregulation resulted in G0-1/S phase arrest, while CCDC144NL-AS1 overexpression led to G0-1/S phase acceleration. Further detection of hallmarks in G1/S phase, including CDK4, Cyclin D1 and p27, indicated that CCDC144NL-AS1 was a vital role in acceleration of G1/S transition in CRC cells. Hence, CCDC144NL-AS1 exerted as a ceRNA, may promote cell proliferation in CRC by accelerating G1/S transition. Even so, this study still has some limitations. In one respect, the deeper mechanism that how CCDC144NL-AS1/miR-363-3p/GALNT7 axis promoted cell proliferation in CRC waits to be excavated. In the other respect, the possible relationship between CCDC144NL-AS1 and tumor metastasis were never detected in this study, which need further investigate.

To conclude, we investigated that CCDC144NLA-AS1 was a novel oncogenic lncRNA in CRC. Then, we demonstrated that CCDC144NL-AS1 could facilitate cell growth and promote cells transition from G0-G1 phase to $S$ phase though miR-363-3p-GALNT7 axis in CRC. The study suggested that CCDC144NL-AS1 may be potential targets of diagnosis and therapies for CRC.

\section{Abbreviations}

CRC: colorectal cancer; lncRNA: long noncoding RNA; siRNA: small interfering RNA; shRNA: small hairpin RNA; CCK-8: Cell Counting Kit-8; Edu: 5-ethynyl-2'-deoxyuridin; FISH: RNA fluorescent in situ hybridization; IHC: Immunohistochemistry; COAD: colon adenocarcinoma; ceRNA: competing endogenous RNA; GALNT7: GalNAc-transferase-7.

\section{Supplementary Material}

Supplementary table.

https://www.jcancer.org/v13p0752s1.pdf

\section{Acknowledgements}

\section{Funding}

This study was supported by Jiangsu Provincial Natural Science Foundation for Basic Research, China (Grant No. BK20201491) and Jiangsu Key Medical Discipline (General Surgery; Grant No. ZDxKA2016005).

\section{Availability of data and materials}

The datasets used and/or analyzed during the current study are available from the corresponding author on reasonable request.

\section{Competing Interests}

The authors have declared that no competing interest exists.

\section{References}

1. Bray F, Ferlay J, Soerjomataram I, Siegel RL, Torre LA, Jemal A. Global cancer statistics 2018: GLOBOCAN estimates of incidence and mortality worldwide for 36 cancers in 185 countries. CA Cancer J Clin. 2018; 68: 394-424.

2. Chen W, Zheng R, Baade PD, Zhang S, Zeng H, Bray F, et al. Cancer statistics in China, 2015. CA Cancer J Clin. 2016; 66: 115-32.

3. Dekker E, Tanis PJ, Vleugels JLA, Kasi PM, Wallace MB. Colorectal cancer. Lancet. 2019: 394: 1467-80.

4. Hashiguchi Y, Muro K, Saito Y, Ito Y, Ajioka Y, Hamaguchi T, et al. Japanese Society for Cancer of the Colon and Rectum (JSCCR) guidelines 2019 for the treatment of colorectal cancer. Int J Clin Oncol. 2020; 25: 1-42.

5. Nagano T, Fraser P. No-nonsense functions for long noncoding RNAs. Cell. 2011; 145: 178-81.

6. Chen LL. Linking Long Noncoding RNA Localization and Function. Trends Biochem Sci. 2016; 41: 761-72.

7. Yao RW, Wang Y, Chen LL. Cellular functions of long noncoding RNAs. Nat Cell Biol. 2019; 21: 542-51.

8. Cheetham SW, Gruhl F, Mattick JS, Dinger ME. Long noncoding RNAs and the genetics of cancer. Br J Cancer. 2013; 108: 2419-25.

9. Gutschner T, Diederichs S. The hallmarks of cancer: a long non-coding RNA point of view. RNA Biol. 2012; 9: 703-19.

10. Mercer TR, Mattick JS. Structure and function of long noncoding RNAs in epigenetic regulation. Nat Struct Mol Biol. 2013; 20: 300-7.

11. Zhu T, Wang Z, Wang G, Hu Z, Ding H, Li R, et al. Long non-coding RNA ZFAS1 promotes the expression of EPAS1 in gastric cardia adenocarcinoma. J Adv Res. 2021; 28: 7-15

12. Ni W, Yao S, Zhou Y, Liu Y, Huang P, Zhou A, et al. Long noncoding RNA GAS5 inhibits progression of colorectal cancer by interacting with and triggering YAP phosphorylation and degradation and is negatively regulated by the m(6)A reader YTHDF3. Mol Cancer. 2019; 18: 143.

13. Wang $\mathrm{Y}, \mathrm{Lu} \mathrm{JH}, \mathrm{Wu} \mathrm{QN}$, Jin $\mathrm{Y}$, Wang DS, Chen YX, et al. LncRNA LINRIS stabilizes IGF2BP2 and promotes the aerobic glycolysis in colorectal cancer. Mol Cancer. 2019; 18: 174.

14. Xu M, Xu X, Pan B, Chen X, Lin K, Zeng K, et al. LncRNA SATB2-AS1 inhibits tumor metastasis and affects the tumor immune cell microenvironment in colorectal cancer by regulating SATB2. Mol Cancer. 2019; 18: 135.

15. Wang Y, Guo B, Xiao Z, Lin H, Zhang X, Song Y, et al. Long noncoding RNA CCDC144NL-AS1 knockdown induces naive-like state conversion of human pluripotent stem cells. Stem Cell Res Ther. 2019; 10: 220.

16. Zhang C, Wu W, Zhu H, Yu X, Zhang Y, Ye X, et al. Knockdown of long noncoding RNA CCDC144NL-AS1 attenuates migration and invasion phenotypes in endometrial stromal cells from endometriosisdagger. Biol Reprod. 2019; 100: 939-49.

17. Fan $\mathrm{H}, \mathrm{Ge} \mathrm{Y}, \mathrm{Ma} \mathrm{X}, \mathrm{Li} \mathrm{Z}$, Shi $\mathrm{L}$, Lin $\mathrm{L}$, et al. Long non-coding RNA CCDC144NL-AS1 sponges miR-143-3p and regulates MAP3K7 by acting as a competing endogenous RNA in gastric cancer. Cell Death Dis. 2020; 11: 521

18. He J, Guan J, Liao S, Wu Z, Liu B, Mo H, et al. Long Noncoding RNA CCDC144NL-AS1 Promotes the Oncogenicity of Osteosarcoma by Acting as a Molecular Sponge for microRNA-490-3p and Thereby Increasing HMGA2 Expression. Onco Targets Ther. 2021; 14: 1-13.

19. Guo L, Peng Y, Meng Y, Liu Y, Yang S, Jin H, et al. Expression profiles analysis reveals an integrated miRNA-lncRNA signature to predict survival in ovarian cancer patients with wild-type BRCA1/2. Oncotarget. 2017; 8: 68483-92.

20. Liu H, Gu X, Wang G, Huang Y, Ju S, Huang J, et al. Copy number variations primed lncRNAs deregulation contribute to poor prognosis in colorectal cancer. Aging (Albany NY). 2019; 11: 6089-108.

21. Li J, Peng W, Yang P, Chen R, Gu Q, Qian W, et al. MicroRNA-1224-5p Inhibits Metastasis and Epithelial-Mesenchymal Transition in Colorectal Cancer by Targeting SP1-Mediated NF-kB Signaling Pathways. Frontiers in oncology. 2020; 10: 294.

22. Zhang Z, Li J, Huang Y, Peng W, Qian W, Gu J, et al. Upregulated miR-1258 regulates cell cycle and inhibits cell proliferation by directly targeting E2F8 in CRC. Cell proliferation. 2018; 51: e12505.

23. Zhang Y, Feng Y, Ji D, Wang Q, Qian W, Wang S, et al. TRIM27 functions as an oncogene by activating epithelial-mesenchymal transition and p-AKT in colorectal cancer. Int J Oncol. 2018; 53: 620-32.

24. Kopp F, Mendell JT. Functional Classification and Experimental Dissection of Long Noncoding RNAs. Cell. 2018; 172: 393-407.

25. Schmitz SU, Grote P, Herrmann BG. Mechanisms of long noncoding RNA function in development and disease. Cell Mol Life Sci. 2016; 73: 2491-509.

26. Kong X, Duan Y, Sang Y, Li Y, Zhang H, Liang Y, et al. LncRNA-CDC6 promotes breast cancer progression and function as ceRNA to target CDC6 by sponging microRNA-215. J Cell Physiol. 2019; 234: 9105-17.

27. Peng WX, Koirala P, Mo YY. LncRNA-mediated regulation of cell signaling in cancer. Oncogene. 2017; 36: 5661-7.

28. Salmena L, Poliseno L, Tay Y, Kats L, Pandolfi PP. A ceRNA hypothesis: the Rosetta Stone of a hidden RNA language? Cell. 2011; 146: 353-8.

29. Tay Y, Rinn J, Pandolfi PP. The multilayered complexity of ceRNA crosstalk and competition. Nature. $2014 \cdot 505 \cdot 344-52$. 
30. Xu M, Chen X, Lin $\mathrm{K}$, Zeng $\mathrm{K}$, Liu $\mathrm{X}, \mathrm{Xu} \mathrm{X}$, et al. IncRNA SNHG6 regulates EZH2 expression by sponging miR-26a/b and miR-214 in colorectal cancer. J Hematol Oncol. 2019; 12: 3.

31. Zhao Y, Du T, Du L, Li P, Li J, Duan W, et al. Long noncoding RNA LINC02418 regulates MELK expression by acting as a ceRNA and may serve as a diagnostic marker for colorectal cancer. Cell Death Dis. 2019; 10: 568.

32. Hou G, Chen H, Yin Y, Pan Y, Zhang X, Jia F. MEL Ameliorates Post-SAH Cerebral Vasospasm by Affecting the Expression of eNOS and HIF1alpha via H19/miR-138/eNOS/NO and H19/miR-675/HIF1alpha. Mol Ther Nucleic Acids. 2020; 19: 523-32.

33. Wang R, Zhou S, Wu P, Li M, Ding X, Sun L, et al. Identifying Involvement of H19-miR-675-3p-IGF1R and H19-miR-200a-PDCD4 in Treating Pulmonary Hypertension with Melatonin. Mol Ther Nucleic Acids. 2018; 13: 44-54

34. Chen DL, Lu YX, Zhang JX, Wei XL, Wang F, Zeng ZL, et al. Long non-coding RNA UICLM promotes colorectal cancer liver metastasis by acting as a ceRNA for microRNA-215 to regulate ZEB2 expression. Theranostics. 2017; 7: 4836-49.

35. Lou W, Ding B, Zhong G, Du C, Fan W, Fu P. Dysregulation of pseudogene/lncRNA-hsa-miR-363-3p-SPOCK2 pathway fuels stage progression of ovarian cancer. Aging (Albany NY). 2019; 11: 11416-39.

36. Wang K, Yan L, Lu F. miR-363-3p Inhibits Osteosarcoma Cell Proliferation and Invasion via Targeting SOX4. Oncol Res. 2019; 27: 157-63.

37. Chang J, Gao F, Chu H, Lou L, Wang H, Chen Y. miR-363-3p inhibits migration, invasion, and epithelial-mesenchymal transition by targeting NEDD9 and SOX4 in non-small-cell lung cancer. J Cell Physiol. 2020; 235: 1808-20.

38. Wang J, Tang Q, Lu L, Luo Z, Li W, Lu Y, et al. LncRNA OIP5-AS1 interacts with miR-363-3p to contribute to hepatocellular carcinoma progression through up-regulation of SOX4. Gene Ther. 2019; 27: 495-504.

39. Dong J, Geng J, Tan W. MiR-363-3p suppresses tumor growth and metastasis of colorectal cancer via targeting SphK2. Biomed Pharmacother. 2018; 105: 922-31.

40. Xie JJ, Li WH, Li X, Ye W, Shao CF. LncRNA MALAT1 promotes colorectal cancer development by sponging miR-363-3p to regulate EZH2 expression. J Biol Regul Homeost Agents. 2019; 33: 331-43.

41. Ghasemi T, Khalaj-Kondori M, Hosseinpour Feizi MA, Asadi P. IncRNA-miRNA-mRNA interaction network for colorectal cancer; An in silico analysis. Comput Biol Chem. 2020; 89: 107370.

42. Wang JB, Chen $\mathrm{XL}$, Han ZB, Wang HW, Wang ZH, Li NN, et al. Long non-coding RNA TP73-AS1 contributes to glioma tumorigenesis by sponging the miR-103a/GALNT7 pathway. Brain Res. 2020; 1741: 146886.

43. Cao $\mathrm{O}$, Wang $\mathrm{N}$, Ren $\mathrm{L}$, Tian J, Yang $\mathrm{S}$, Cheng $\mathrm{H}$. miR-125a-5p post-transcriptionally suppresses GALNT7 to inhibit proliferation and invasion in cervical cancer cells via the EGFR/PI3K/AKT pathway. Cancer Cell Int. 2020; 20: 117.

44. Li Y, Zeng C, Hu J, Pan Y, Shan Y, Liu B, et al. Long non-coding RNA-SNHG7 acts as a target of miR-34a to increase GALNT7 level and regulate $\mathrm{PI} 3 \mathrm{~K} / \mathrm{Akt} / \mathrm{mTOR}$ pathway in colorectal cancer progression. J Hematol Oncol. 2018; 11: 89

45. Niu JT, Zhang LJ, Huang YW, Li C, Jiang N, Niu YJ. MiR-154 inhibits the growth of laryngeal squamous cell carcinoma by targeting GALNT7. Biochem Cell Biol. 2018; 96: 752-60.

46. $\mathrm{Li} \mathrm{W}, \mathrm{Ma} \mathrm{H}$, Sun J. MicroRNA34a/c function as tumor suppressors in Hep2 laryngeal carcinoma cells and may reduce GALNT7 expression. Mol Med Rep. 2014; 9: 1293-8.

47. Otto T, Sicinski P. Cell cycle proteins as promising targets in cancer therapy. Nat Rev Cancer. 2017; 17: 93-115.

48. Bertoli C, Skotheim JM, de Bruin RA. Control of cell cycle transcription during G1 and S phases. Nat Rev Mol Cell Biol. 2013; 14: 518-28. 